\title{
The Principal Principle does not imply the Principle of Indifference
}

\author{
Richard Pettigrew* \\ forthcoming in The British Journal for the Philosophy of Science
}

September 12, 2017

All Bayesian epistemologists agree on two claims. The first, which we might call Precise Credences, says that an agent's doxastic state at a given time $t$ in her epistemic life can be represented by a single credence function $P_{t}$, which assigns to each proposition $A$ about which she has an opinion a precise numerical value $P_{t}(A)$ that is at least 0 and at most $1 . P_{t}(A)$ is the agent's credence in $A$ at $t$. It measures how strongly she believes $A$ at $t$, or how confident she is at $t$ that $A$ is true. The second point of agreement, which is typically known as Probabilism, says that an agent's credence function at a given time should be a probability function: that is, for all times $t, P_{t}(\top)=1$ for any tautology $\top, P_{t}(\perp)=0$ for any contradiction $\perp$, and $P_{t}(A \vee B)=P_{t}(A)+P_{t}(B)-P_{t}(A B)$ for any propositions $A$ and $B$.

So Precise Credences and Probabilism form the core of Bayesian epistemology. But, beyond these two norms, there is little agreement between its adherents. Bayesian epistemologists disagree along (at least) two dimensions. First, they disagree about the correct norms concerning updating on evidence learned with certainty - some say there are diachronic norms concerning how an agent should in fact update; others say there are only synchronic norms concerning how an agent should plan to update; and others think there are no norms concerning updating at all. Second, they disagree about the stringency of the synchronic norms that don't concern updating. Our concern here is with the latter. Two candidates norms of this sort: the Principal Principle, which says how an agent's credences in propositions concerning the objective chances should relate to her credences in other propositions (Lewis, 1980); and the Principle of Indifference, which says, roughly, that an agent with no evidence should divide

\footnotetext{
*Acknowledgments: I am very grateful to the following people for very valuable discussion during the drafting of this paper: Catrin Campbell-Moore, Graeme Forbes, Casey Hart, Jim Hawthorne, Jason Konek, Juergen Landes, Jan-Willem Romeijn, Mike Titelbaum, Christian Wallmann, Jon Williamson, Leszek Wronski, and two anonymous referees for this journal.
} 
her credences equally over all possibilities (Keynes, 1921; Carnap, 1950; Jaynes, 2003; Williamson, 2010; Pettigrew, 2014). Those we might call Radical Subjective Bayesians (or RSBs) adhere to Precise Credences and Probabilism, but reject the Principal Principle and the Principle of Indifference. Those we might call Moderate Subjective Bayesians (or MSBs) adhere to Precise Credences, Probabilism, and the Principal Principle, but they reject the Principle of Indifference. And the Objective Bayesians (or OBs) accept all of the principles. The following table summarises the situation:

\begin{tabular}{|l|c|c|c|}
\hline & RSB & MSB & OB \\
\hline Precise Credences & $\checkmark$ & $\checkmark$ & $\checkmark$ \\
Probabilism & $\checkmark$ & $\checkmark$ & $\checkmark$ \\
Principal Principle & $\times$ & $\checkmark$ & $\checkmark$ \\
Principle of Indifference & $\times$ & $\times$ & $\checkmark$ \\
\hline
\end{tabular}

In a recent paper, Hawthorne et al. (2015) (henceforth, HLWW) argue that MSB is an unstable position, because the Principal Principle entails the Principle of Indifference. Thus, it is inconsistent to accept the former and reject the latter. We must either reject the Principal Principle, as the RSB does, or accept it together with the Principle of Indifference, as the OB does.

Notoriously, as Lewis originally stated it, the Principal Principle includes an admissibility condition (Lewis, 1980, 266-7). Equally notoriously, Lewis did not provide a precise account of this condition, thereby leaving his formulation of the principle similarly imprecise. HLWW do not give a precise account either, but they do appeal to two principles concerning admissibility, which they call Condition 1 and Condition 2 (Hawthorne et al., $2015,124)$. There are two ways of reading their argument, depending on how you understand the status of Conditions 1 and 2. Reading 1: The correct account of admissibility is determined independently of these two principles, and yet these two principles follow from that correct account. Reading 2: The correct account of admissibility is determined in part by these two principles, so that the principles follow from that account but only because the correct account is constrained so that it must satisfy them. HLWW then show that, given an account of admissibility on which Conditions 1 and 2 hold, the Principal Principle entails the Principle of Indifference (Hawthorne et al., 2015, Proposition 2). I will argue that, on either reading of the argument, it fails. I will argue in section 4 that there is a plausible account of admissibility on which Conditions 1 and 2 are false. That defeats the first reading of the argument. I will then argue in section 5 that the intuitions that lead us to assent to Condition 2 also lead us to assent to other very closely related principles that are inconsistent with Condition 2. This, I claim, casts doubt on the reliability of those intuitions, and thus removes our justification for Condition 2. This defeats the second reading 
of the HLWW argument. Thus, the argument fails. Moderate Subjective Bayesianism is a coherent position.

\section{Introducing the Principal Principle}

We begin by introducing the Principal Principle. ${ }^{1}$ To aid our statement, let me introduce a piece of notation. Given a proposition $A$, a time $t$, and a real number $0 \leq x \leq 1$, let $C_{x}^{A, t}$ be the following proposition: The objective chance of $A$ at $t$ is $x$. And we will let $P_{0}$ be the credence function of our agent at the very beginning of her epistemic life - when she is, as Lewis would say, a superbaby; that is, she is not yet in receipt of any evidence. Then, as HLWW formulate the Principal Principle, it says this:

HLWW's Principal Principle Suppose $A, E$ are propositions, $t$ is a time, and $0 \leq x \leq 1$. Then it should be the case that

$$
P_{0}\left(A \mid C_{x}^{A, t} E\right)=x
$$

providing (i) $P_{0}\left(C_{x}^{A, t} E\right)>0$, and (ii) $E$ is admissible for $A$ at $t$.

In this version, the principle applies to an agent only at the beginning of her epistemic life; it governs her initial credence function. In this situation, the principle says, her credence in a proposition $A$ conditional on the conjunction of some proposition $E$ and a chance proposition that says that the chance of $A$ at $t$ is $x$ should be $x$, providing the conditional probability is well-defined and $E$ is admissible for $A$ at time $t$.

The motivation for the admissibility condition is this. Suppose $E$ entails $A$. Then we surely don't want to demand that $P_{0}\left(A \mid C_{x}^{A, t} E\right)=x$. After all, if $x<1$, then such a demand would conflict with Probabilism, since it is a consequence of Probabilism that, if $E$ entails $A$, then $P_{0}\left(A \mid C_{x}^{A, t} E\right)=1$. Thus, we must at least restrict the Principal Principle so that it does not apply when $E$ entails $A$. But there are other cases in which the Principal Principle should not be imposed, even if such an application would not be outright inconsistent with other norms such as Probabilism. For instance, suppose that $E$ entails that the chance of $A$ at some later time $t^{\prime}>t$ is $x^{\prime} \neq x$. Then, again, we don't want to require that $P_{0}\left(A \mid C_{x}^{A, t} E\right)=x$,

\footnotetext{
${ }^{1}$ Now, as Lewis himself noted, the Principal Principle has implausible consequences when the chances are self-undermining - that is, when the chances assign a positive probability to outcomes in which the chances are different. This happens, for instance, for Lewis' own favoured account of chance, the Humean account or Best System Analysis. This lead to reformulations of the Principal Principle, such as Thau's and Hall's New Principle (Lewis, 1994; Thau, 1994; Hall, 1994) and Ismael's General Recipe (Ismael, 2008). HLWW say nothing explicitly about whether or not chances are self-undermining. But, since they are interested in investigating the Principal Principle and not the New Principle or the General Recipe, I take them to assume that chances are not self-undermining. I will do likewise.
} 
because information about later chances trumps information about earlier chances. The moral is this: if $E$ contains information about $A$ that trumps or overrides the information provided by the proposition, $C_{x}^{A, t}$, which says that the chance of $A$ at $t$ is $x$, then it is inadmissible for $A$ at $t$. Clearly any proposition that logically entails $A$ provides such overriding information; and so does a proposition that entails something about the chance of $A$ at a later time. So much for propositions that are inadmissible. Are there any we can be sure are admissible for $A$ at $t$ ? According to Lewis, there are, namely, propositions solely concerning time $t$ or earlier times $t^{\prime}<t$. Thus, Lewis does not give a precise account of admissibility: he gives a heuristic - $E$ is admissible for $A$ at $t$ if $E$ does not provide information about $A$ that overrides the information contained in propositions about the chance of $A$ at $t-$ and he gives examples of propositions that do and do not provide such information - I've recalled some of Lewis' examples here.

\section{Introducing the Principle of Indifference}

The HLWW argument seeks to establish that the Principle of Indifference follows from the Principal Principle. We have met the latter; now, let's meet the former. In fact, the version of the Principle of Indifference that HLWW consider is rather weaker than the usual version of that norm. According to the usual version, an agent with no evidence should divide her credences equally across all possibilities. If I have credences in two atomic propositions, $F$ and $G$, and all Boolean combinations of them, then the possibilities in question are $F G, F \bar{G}, \bar{F} G$, and $\overline{F G}{ }^{2}$. Thus, according to the usual version of the Principle of Indifference (together with Probabilism), if I have no evidence whatsoever, I should assign credences as follows:

$$
P_{0}(F G)=P_{0}(F \bar{G})=P_{0}(\bar{F} G)=P_{0}(\bar{F} \bar{G})=\frac{1}{4}
$$

Instead, the version of the Principle of Indifference that appears in the HLWW argument just says this:

HLWW's Principle of Indifference Suppose $F$ is an atomic proposition. Then it should be the case that

$$
P_{0}(F)=\frac{1}{2}
$$

\footnotetext{
${ }^{2}$ According to HLWW, a proposition is atomic iff it is "not logically complex" (Hawthorne et al., 2015, 124). They give the following examples: "Fido is exploding" and "The sun will rise tomorrow". Thus, atomic propositions that ascribe a predicate to a subject. They are the propositions that we would render as atomic formulae were we to create a language for the world in first-order logic. As we will see below, the notion of an atomic formulae plays an important role in HLWW's argument. On the basis of this loose characterization, we might wonder whether the notion can bear that weight - after all, it is not clear that there is always a determinate fact of the matter whether a proposition is logically complex or logically simple. I will not pursue this objection here.
} 
It is clear that this does not entail the usual version. In the case above, in which I have credences in atomic propositions $F$ and $G$ and their Boolean combinations, it demands that

$$
P_{0}(F)=P_{0}(G)=\frac{1}{2}
$$

but nothing stronger. It imposes no constraints on my credences in $F G$ and $F \bar{G}$ other than that they should sum to $\frac{1}{2}$; and it imposes no constraints on my credences in $F G$ and $\bar{F} G$ other than that they should sum to $\frac{1}{2}$. However, it is nonetheless strong enough that the Radical Subjective Bayesian (RSB) will deny it. They claim that credences even in atomic propositions are unconstrained at the beginning of an agent's epistemic life.

\section{The HLWW argument}

The HLWW argument turns on a mathematical theorem:

Theorem 1 ((Hawthorne et al., 2015)) Suppose $P$ is a probability function. If there is $0<x<1$ such that

(i) $P(A \mid X F)=x$

(ii) $P(A \mid X(A \leftrightarrow F))=x$

(iii) $P(F \mid X)=P(F)$

then

(iv) $P(F)=\frac{1}{2}$.

How can we use this to establish HLWW's Principle of Indifference on the basis of Lewis' Principal Principle? Well, notice first that (iv) is the condition imposed on atomic propositions by HLWW's Principle of Indifference. Thus, if, for any atomic proposition $F$, we can find $A, X$, and $0<x<1$ such that (i), (ii), and (iii) hold, then we can establish HLWW's Principle of Indifference. This is where Lewis' Principal Principle enters. Let $F$ be an atomic proposition. First, we find a time $t$ and a proposition $A$ (not necessarily atomic) such that: (a) the truth value of $A$ is not fixed by $t$; and (b) our agent's evidence gives no information about the connection between $F$ and $A$. Then pick any $0<x<1$ and let $X$ be the proposition $C_{x}^{A, t}$, which says that the objective chance of $A$ at $t$ is $x$. Then, according to HLWW, (i), (ii), and (iii) hold. First, they appeal to what they call Condition 1 (which I paraphrase here):

Condition 1 If your evidence at $t$ gives no information about the connection between $A$ and $F$, then $F$ is admissible for $A$ at $t$ (where $F$ is an atomic proposition). 
Together with Lewis' Principal Principle, this gives clause (i) $-P(A \mid X F)=$ $P\left(A \mid C_{x}^{A, t} F\right)=x$. Second, they appeal to what they call Condition 2 (again, paraphrased here):

Condition 2 If your evidence at $t$ gives no information about the connection between $A$ and $F$, then $A \leftrightarrow F$ is admissible for $A$ at $t$ (where $F$ is an atomic proposition).

Together with Lewis' Principal Principle, this gives clause (ii) $-P(A \mid X(A \leftrightarrow$ $F))=P\left(A \mid C_{x}^{A, t}(A \leftrightarrow F)\right)=x$. Finally, they appeal to what I will call Condition 3:

Condition 3 If your evidence at $t$ gives no information about the connection between $A$ and $F$, then your credence function should treat $F$ and $C_{x}^{A, t}$ as evidentially irrelevant to one another (for any probability $x$ ); that is, your credence function should render $F$ and $C_{x}^{A, t}$ probabilistically independent.

This gives (iii). Thus, together with Lewis' Principal Principle, Conditions 1, 2, and 3 give clauses (i), (ii), and (iii). Thus, they give (iv). And so they secure for us HLWW's Principle of Indifference.

The upshot: in order to derive HLWW's Principle of Indifference from Lewis' Principal Principle, we need three further ingredients: Conditions 1,2 , and 3 . Conditions 1 and 2 concern admissibility. In the following two sections we consider two readings of these conditions - on the first, they are intended to be justified on the basis of the correct account of admissibility (section 4); on the second, they are constraints on the correct account of admissibility, and such an account is justified in part by showing that it satisfies them (section 5).

\section{Reading 1: Admissibility justifies Conditions 1 and 2}

On this reading, we have an account of admissibility that we obtain independently of considering Conditions 1 and 2, and that account of admissibility entails those conditions. I will object to the argument read in this way by presenting an account of admissibility, demonstrating its strengths, and showing that Conditions 1 and 2 are false according to that account.

Above, we stated the Principal Principle as follows:

\section{HLWW's Principal Principle}

$$
P_{0}\left(A \mid C_{x}^{A, t} E\right)=x,
$$

providing (i) $P_{0}\left(C_{x}^{A, t} E\right)>0$, and (ii) $E$ is admissible for $A$ at $t$. 
Now suppose we make the following assumption about admissibility:

Contemporaneous Chance Admissibility Propositions that solely concern the objective chances at $t$ are admissible for any proposition at $t$.

Thus, for instance, $P_{0}\left(A \mid C_{x}^{A, t} C_{y}^{B, t}\right)=x$, providing $P_{0}\left(C_{x}^{A, t} C_{y}^{B, t}\right)>0$, which also ensures that $C_{x}^{A, t}$ and $C_{y}^{B, t}$ are compatible.

Now suppose that, if $c h$ is a probability function defined over all the propositions about which the agent has an opinion, $C_{c h}^{t}$ is the proposition that says that the objective chances at $t$ are given by $c h{ }^{3}$ Then it follows from HLWW's Principal Principle and Contemporaneous Chance Admissibility that $P_{0}\left(A \mid C_{c h}^{t}\right)=\operatorname{ch}(A) \cdot{ }^{4}$ But it also follows from this that: ${ }^{5}$

\section{Levi's Principal Principle (Bogdan, 1984; Hall, 2004; Pettigrew,} 2012)

$$
P_{0}\left(A \mid C_{c h}^{t} E\right)=\operatorname{ch}(A \mid E),
$$

providing $P_{0}\left(C_{c h}^{t} E\right), \operatorname{ch}(E)>0$.

This is a version of the Principal Principle that makes no mention of admissibility. From it, something close to HLWW's Principal Principle follows: Let $C_{x}^{A \mid E, t}$ be the following proposition: The objective chance of $A$ conditional on $E$ at $t$ is $x$. Then Levi's Principal Principle entails that

$$
P_{0}\left(A \mid C_{x}^{A \mid E, t} E\right)=x
$$

whenever $P_{0}\left(C_{x}^{A \mid E, t} E\right)>0$. Thus, instead of setting your conditional credence in $A$ given that $c h$ is the objective chance function to $c h(A)$, you bring ch up to speed with your evidence $E$, and set your credence to $\operatorname{ch}(A \mid E)$ instead.

A striking feature of Levi's Principal Principle is that it does not appeal to the notion of admissibility at any point. It applies equally when the proposition $E$ is not admissible in Lewis' sense. Nonetheless, it does suggest a precise account of admissibility.

\footnotetext{
${ }^{3}$ This might seem a demanding assumption. Surely it is possible for an agent to have a rational credence in a proposition to which it is not possible to attach an objective chance. In fact, it is not as demanding as you might expect. To those propositions to which it seems unnatural to assign an objective chance, the chance function assigns its truth value -0 if the proposition is false, or 1 if the proposition is true.

${ }^{4}$ After all, $C_{c h}^{t}$ is simply a conjunction of propositions about the objective chances at $t$. One of these is $C_{x}^{A, t}$, where $\operatorname{ch}(A)=x$. And the others are all admissible.

${ }^{5}$ Proof.

$$
P_{0}\left(A \mid C_{c h}^{t} E\right)=\frac{P_{0}\left(A E \mid C_{c h}^{t}\right)}{P_{0}\left(E \mid C_{c h}^{t}\right)}=\frac{\operatorname{ch}(A E)}{\operatorname{ch}(E)}=\operatorname{ch}(A \mid E)
$$

as required.
} 
Levi-Admissibility $E$ is Levi-admissible for $A$ at $t$ iff, for all possible chance functions $\operatorname{ch}$ at $t$, if $\operatorname{ch}(E)>0$, then $\operatorname{ch}(A \mid E)=\operatorname{ch}(A)$.

That is, on this account $A$ is admissible for $E$ at a time if every possible chance function at that time renders $A$ and $E$ stochastically independent. Three points are worthy of note:

(1) All propositions providing information about the chance of $A$ at a later time $t^{\prime}>t$, or information about the truth value of $A$, are Leviinadmissible for $A$ at $t$, since $A$ will be stochastically dependent on such propositions according to some possible chance functions at $t$. So this account of admissibility agrees with the examples of clearly inadmissible propositions that we gave above.

(2) All propositions solely about time $t$ or earlier times $t^{\prime}<t$ are Leviadmissible for $A$ at $t$, since all such propositions will either be true at $t$ or false at $t$, and thus will be assigned chance 1 or 0 accordingly by all possible chance functions at $t{ }^{6}$ So this account of admissibility agrees with the examples of clearly admissible propositions that we gave above.

(3) If $E$ is Levi-admissible for $A$ at $t$, then $P_{0}\left(A \mid C_{x}^{A, t} E\right)=P_{0}\left(A \mid C_{x}^{A \mid E, t} E\right)=$ $x$. That is, HLWW's Principal Principle follows from Levi's version if we understand the notion of admissibility as Levi-admissibility.

Taken together, (1), (2), and (3) entail that Levi-admissibility has all of the features that Lewis wished admissibility to have.

Now, although Levi's account of admissibility recovers Lewis' examples, it might seem too demanding. Suppose, for instance, that $A$ is a proposition concerning the toss of a coin in Quito tomorrow - it says that it will lands heads - while $E$ is a proposition concerning tomorrow's weather in Addis Ababa - it says that it will rain. Then, intuitively, $E$ is admissible

\footnotetext{
${ }^{6}$ For any probability function $c h$ and any proposition $A$ and $E$, if $\operatorname{ch}(E)=0$ or $\operatorname{ch}(E)=1$, then $A$ and $E$ are stochastically independent. Note, however, that some philosophers deny that past propositions have chance 0 or 1 - that is, they take some past propositions still to be chancy (Hoefer, 2007; Frigg \& Hoefer, 2010; List \& Pivato, 2015). This is no problem for Levi's Principal Principle nor for Levi-admissibility. It just means there's no guarantee that past events are admissible. But someone who thinks that past events are still chancy is unlikely to think that propositions that say which way those events turn out will always be admissible. However, even amongst philosophers who take all past propositions to have chance 0 or 1 , there are some who take some past propositions to be inadmissible evidence. Thus, for them, (2) is not a desiderata for an account of admissibility. For instance, Nissan-Rozen (2017) claims that there are cases in which a proposition $E$ that is true at $t^{\prime}$ is inadmissible for $A$ at a later time $t>t^{\prime}$ because $E$ provides an explanation of $A$, and propositions that provide explanations contain information that overrides the information provided by the chances. I side with Lewis against Nissan-Rozen on this, but it would take me too far afield to address the issue here. For a valuable discussion, see (Joyce, 2007, 199). Thanks to an anonymous referee for urging me to make these controversies clear.
} 
for $A$ today. But $E$ is not Levi-admissible for $A$ today. After all, we are considering an agent at the beginning of her epistemic life. And so there are certainly possible chance functions - probability functions that, for all she knows, give the objective chances today - that do not render $E$ and $A$ stochastically independent.

However, in fact, on closer inspection, the Levi-admissibility verdict is exactly right. Consider my credence in $A$ conditional on $E$ and the chance hypothesis $C_{0.5}^{A, t}$, which says that the coin in Quito is fair and so the unconditional chance of $A$ today $(t)$ is 0.5 . Amongst the chance functions that are epistemically possible for me, some make $E$ irrelevant to $A$, some make it positively relevant to $A$ and some make it negatively relevant to $A$. Indeed, we might suppose that the possible chances of $A$ conditional on $E$ run the whole gamut of values from 0 to 1 . In that case, surely we don't want to say that $E$ is admissible for $A$ and thereby impose, via the Principal Principle, the demand that our agent's credence in $A$ conditional on $E$ and $C_{0.5}^{A, t}$ is 0.5 . After all, if I choose to place most of my initial credence on the chance hypotheses on which $E$ is positively relevant to $A$, then my credence in $A$ conditional on $E$ and $C_{0.5}^{A, t}$ should not be 0.5 - it should be something greater than 0.5 . If I choose to place most of my initial credence on the chance hypotheses on which $E$ is negatively relevant to $A$, then my credence in $A$ conditional on $E$ and $C_{0.5}^{A, t}$ should not be 0.5 - it should be something less than 0.5 . Of course, we might think that it is irrational for our agent, a superbaby with no evidence one way or the other, to favour the positive relevance hypotheses over those that posit neutral relevance and negative relevance. We might think that she should spread her credences equally over all of the possibilities, in which case their effects will cancel out, and her credence in $A$ conditional on $E$ and $C_{0.5}^{A, t}$ will indeed be 0.5 . But of course to do this is to assume the Principle of Indifference and beg the question.

Another possible objection: Levi's Principal Principle is all well and good in theory, but it is not applicable. Suppose we are interested in a proposition $A$; and we have collected evidence $E$. How might we apply Levi's Principal Principle in order to set our credence in $A$ ? In the case of Lewis' version of the principle, we need only know the chance of $A$ and the fact that $E$ is admissible for $A$, and we often know both of these. But, in order to apply Levi's version, we must know the chance of $A$ conditional on our evidence $E$. And, at least for large and varied bodies of evidence, we never know this. Or so the objection goes.

But the objection fails. In fact, Levi's Principal Principle may be applied in those cases. You don't have to know the chance of $A$ conditional on $E$ in order to set your credence in $A$ when you have evidence $E$. You simply have to have opinions about the different possible values that that conditional chance might take. You then apply Levi's Principal Principle, 
together with the Law of Total Probability, which jointly entail that your credence in $A$ given $E$ should be your expectation of the chance of $A$ given $E$. Of course, neither Levi's Principal Principle nor the Law of Total Probability will tell you how to set your credences in the different possible values that the conditional chance of $A$ given $E$ might take. But that's not a problem for the Moderate Subjective Bayesian, who doesn't expect her evidence to pin down a unique credal response. Only the Objective Bayesian would expect that. You pick your probability distribution over those possible conditional chance values and Levi's Principal Principle does the rest via the Law of Total Probability.

Here's another feature of Levi-admissibility that may at first seem to tell against it: it renders the admissibility relation symmetric. Thus, according to Levi-admissibility, $E$ is admissible for $A$ at time $t$ iff $A$ is admissible for $E$ at $t$. And, equivalently, $E$ is inadmissible for $A$ at $t$ iff $A$ is inadmissible for $E$ at $t$. And yet the examples that we often give to motivate the notion of admissibility might lead us to think that the notion is not symmetric: we take past propositions to be admissible for future ones, but we don't say explicitly that future propositions are admissible for past ones. Nonetheless, Levi-admissibility entails that they are. However, once again, I think Leviadmissibility delivers the correct answer. Suppose we have three times $t<t^{\prime}<t^{\prime \prime}$. Suppose the truth value of $E$ will be settled by the earliest time $t$, but the truth value of $A$ will only be settled by the latest time $t^{\prime \prime}$. And suppose that we are considering our credences conditional on a supposition about the chances at the intermediate time $t^{\prime}$. Then, intuitively, $E$ is guaranteed to be admissible for $A$ at $t^{\prime}$. And Levi-admissibility agrees. Less intuitively, according to Levi-admissibility, $A$ is guaranteed to be admissible for $E$ at $t^{\prime}$. But in fact that is right. After all, at $t^{\prime}$ the truth value of $E$ is fixed. So, according to whichever hypothesis about the chances at $t^{\prime}$ we are considering, its chance at $t^{\prime}$ is 0 or 1 . So, under the supposition of that hypothesis, our credence in $E$ should be 0 or 1 . So, learning $A$ can do nothing to change our credence in $E$. And thus, $A$ is admissible for $E$ at $t^{\prime}$. And Levi-admissibility agrees.

I conclude, then, that Levi-admissibility is at least a plausible candidate account of admissibility. We come to it by adding some minimal assumptions about the admissibility of contemporaneous chance hypotheses, deriving a new version of the Principal Principle (namely, Levi's), and then looking to the account of admissibility that version of the principle suggests. And we have seen that it saves the examples of intuitively admissible and inadmissible propositions that Lewis presented. What's more, we can rebuff three possible objections to it. We now show that Conditions 1 and 2 do not follow from it.

Recall Conditions 1 and 2 from above:

Condition 1 If your evidence at $t$ gives no information about 
the connection between $A$ and $F$, then $F$ is admissible for $A$ at $t$ (where $F$ is an atomic proposition).

Condition 2 If your evidence at $t$ gives no information about the connection between $A$ and $F$, then $A \leftrightarrow F$ is admissible for $A$ at $t$ (where $F$ is an atomic proposition).

Do they follow from the account of admissibility as Levi-admissibility? It is easy to see that they don't. After all, if your evidence gives no information about the connection between $A$ and $F$, that means that it doesn't rule out possible objective chance functions on which $F$ is positively relevant to $A$ nor functions on which $F$ is negatively relevant to $A$. If it were to rule these out, that would mean that it did contain information about the connection between them. Thus, $F$ is not Levi-admissible for $A$. And similarly for $A \leftrightarrow$ $F$. If your evidence gives no information about the connection between $A$ and $F$, it leaves open the possibility that $A \leftrightarrow F$ is positively relevant to $A$ and possibility that it is negatively relevant, and the possible objective chance functions that witness these possibilities also show that $A \leftrightarrow F$ is not Levi-admissible for $A$.

Thus, given the plausible account of admissibility as Levi-admissibility, Conditions 1 and 2 are false and the HLWW argument fails.

\section{Reading 2: Conditions 1 and 2 constrain Admissi- bility}

Of course, one philosopher's modus ponens is another's modus tollens. That Conditions 1 and 2 are incompatible with the account of admissibility as Levi-admissibility might lead me to reject the former; but it might lead you to reject the latter. Indeed, you might take Conditions 1 and 2 to be constraints on admissibility - any candidate account of admissibility must vindicate them, since the intuitions that give rise to them are as strong as those that tell us that propositions solely about the past must be admissible evidence for any proposition about the future, whereas facts about the future chances of a proposition are often inadmissible evidence for that proposition. Or so you claim. Alternatively, you might hold that Conditions 1 and 2 are not intended to be a consequence of the Principal Principle, but rather plausible extra principles that, when combined with the Principal Principle, entail the Principle of Indifference. Of course, in that situation you couldn't very well claim that the Principal Principle on its own entails the Principle of Indifference, but you might offer the Principal Principle + Condition $1+$ Condition 2 as premises in a new argument for the Principle of Indifference. In either case, you need to claim that there are good independent reasons in favour of Conditions 1 and $2-$ good enough 
reasons to prefer them to the account of admissibility as Levi-admissibility; or good enough reasons to accept Conditions 1 and 2 as premises in an argument for the Principle of Indifference if you are not already convinced of the latter. Are there such reasons? I think not.

I will raise an objection against our justification for Condition 2. First, let us consider why we might take Condition 2 to be plausible; let us think of where its intuitive appeal might lie. The idea seems to be this: When I learn that two propositions, $A$ and $F$, are equivalent - that is, when I learn the biconditional $A \leftrightarrow F-$ there are many ways I might respond. I might retain my prior credence in $A$ and bring my credence in $F$ into line with that. Or I might retain my prior credence in $F$ and bring my credence in $A$ into line with that. Or I might do many other things. Condition 2 says that, in one particular sort of situation, I should retain my prior credence in $A$ and bring my credence in $F$ into line with that. The sort of situation is this: I have very strong evidence supporting my credence in $A$ because I know its chance; I have no evidence concerning $F$; and I have no evidence concerning the connection, if any, between $A$ and $F$. In this situation, the reasoning seems to go, I should stick with the credence for which I have strongest evidential support, namely, $A$. Now, on the face of it, this seems like a reasonable constraint on our response to evidence. It says, essentially, that a credence formed in response to stronger evidence should be more resilient than a credence formed in response to weaker evidence. And, as a limiting case, a credence formed in response to strong evidence, such as evidence about the chances, should be maximally resilient when compared to a credence formed in response to no evidence.

The problem is that such reasoning leads to contradictions. Let's see how. Suppose that $G$, like $F$, is an atomic proposition. Then it is easy to suppose that, just as our evidence provides no information about the connection between $A$ and $F$, it similarly provides no information about the connection between $A$ and $F G$, nor any information about the connection between $A$ and $F \bar{G}$. For instance, suppose Astrid, Felix, and Garfield are cats. Suppose $A$ says that Astrid is black, $F$ says that Felix is black, and $G$ says that Garfield is black. Then it might well be that your evidence provides no information about the connection between $A$ and $F$, no information about the connection between $A$ and $F G$, and no information about the connection between $A$ and $F \bar{G}$. If we follow the reasoning used to justify Condition 2 above, we can therefore justify the following three constraints on our credence function $P$ :

(a) $P\left(A \mid C_{x}^{A, t}(A \leftrightarrow F)\right)=x$

(b) $P\left(A \mid C_{x}^{A, t}(A \leftrightarrow F G)\right)=x$

(c) $P\left(A \mid C_{x}^{A, t}(A \leftrightarrow F \bar{G})\right)=x$ 
Unfortunately, however, when $0<x<1$, (a), (b), and (c) are inconsistent constraints on a probability function. ${ }^{7}$ The problem is that, while Condition 2 restricts its application to biconditionals $A \leftrightarrow F$ in which $F$ is atomic, the motivation just given for it justifies the more general principle that does not impose this proviso. That is, it justifies what we might call Condition $2^{+}$:

Condition $2^{+}$If your evidence at $t$ gives no information about the connection between $A$ and $F$, then $A \leftrightarrow F$ is admissible for $A$ at $t$ (for any proposition $F$ ).

And the inconsistency of (a), (b), and (c) shows that Condition $2^{+}$is inconsistent, since it entails all three of them. But if we offer a justification for a principle, and our principle in fact establishes a more general but inconsistent principle if it establishes anything at all, then our putative justification fails - it does not justify the original principle. For instance, you cannot justify an axiom of set theory by appealing to Frege's Basic Law V, since Basic Law $\mathrm{V}$ entails a series of axioms that are mutually inconsistent.

To resist this objection, HLWW must say why Condition 2 is true, while Condition $2^{+}$is false. They must tell us what is so special about atomic propositions. They must say why rationality requires that $P\left(A \mid C_{x}^{A, t}(A \leftrightarrow\right.$ $B))=x$ when $B$ is atomic, but not when $B$ is not. Here is one attempt. Suppose $A, F$, and $G$ are atomic. Then $A$ is true at half of all the possible worlds; and $A$ is true at half of the possible worlds at which $A \leftrightarrow F$ is true. However, while $A$ is true at half of all the possible worlds, it is only true at one quarter of the worlds at which $A \leftrightarrow F G$ is true, and it is only true at one quarter of the worlds at which $A \leftrightarrow F \bar{G}$ is true. Thus, the response goes, learning $A \leftrightarrow F G$ or learning $A \leftrightarrow F \bar{G}$ gives me information about $A$ in a way that learning $A \leftrightarrow F$ does not. The problem with this response is that it ties the information given by one proposition $B$ about another $C$ to the difference between the proportion of $C$-worlds amongst all worlds and the proportion of $C$-worlds amongst $B$-worlds. But that is only reasonable if you divide credences equally over all worlds. And demanding that is simply assuming the Principle of Indifference. So the response begs the question at issue.

\footnotetext{
${ }^{7}$ Let $Q(-)=P\left(-\mid C_{x}^{A, t}\right)$. Now, by the ratio definition of conditional probability and the axioms of probability, if $Q(A \mid A \leftrightarrow X)=x$, then $Q(A X)=x[Q(A X)+Q(\bar{A} \bar{X})]$. Thus:

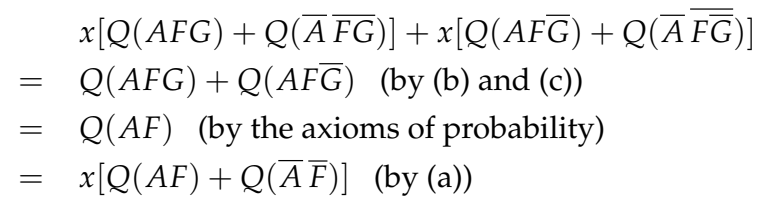

And this gives:

$$
Q(\bar{A} \bar{F} \vee \bar{A} \bar{G})+Q(\bar{A} \bar{F} \vee \bar{A} G)=Q(\bar{A} \bar{F})
$$

which implies $Q(\bar{A} \bar{F})=Q(\bar{A} \bar{G})=Q(\bar{A} G)=0$. Thus, $Q(\bar{A})=0$, and $Q(A)=1$. 


\section{Conclusion}

The HLWW argument purports to show that the Principal Principle entails the Principle of Indifference. But it fails on both of the two readings described above. On the first reading, the crucial premises in the argument, Conditions 1 and 2, are justified by the correct account of admissibility. But we saw that a very plausible candidate account of admissibility in fact renders them false. On the second reading, Conditions 1 and 2 constrain the correct account of admissibility. However, as we saw, the natural justification for Condition 2 in fact entails a stronger principle, Condition $2^{+}$, which is inconsistent. This undermines the justification for Condition 2. The logical space is therefore safe once again for Moderate Subjective Bayesians, that is, those who accept Precise Credences, Probabilism, and the Principal Principle, but who deny the Principle of Indifference.

\section{References}

Bogdan, R. (Ed.) (1984). Henry E. Kyburg, Jr. and Isaac Levi. Dordrecht: Reidel.

Carnap, R. (1950). Logical Foundations of Probability. Chicago: University of Chicago Press.

Frigg, R., \& Hoefer, C. (2010). Determinism and Chance from a Humean Perspective. In F. Stadler (Ed.) The Present Situation in the Philosophy of Science. Springer.

Hall, N. (1994). Correcting the Guide to Objective Chance. Mind, 103, 505-518.

Hall, N. (2004). Two Mistakes About Credence and Chance. Australasian Journal of Philosophy, 82(1), $93-111$.

Hawthorne, J., Landes, J., Wallman, C., \& Williamson, J. (2015). The Principal Principle Implies the Principle of Indifference. The British Journal for the Philosophy of Science.

Hoefer, C. (2007). The Third Way on Objective Probability: A Sceptic's Guide to Objective Chance. Mind, 116(463), 549-596.

Ismael, J. (2008). Raid! Dissolving the Big, Bad Bug. Noûs, 42(2), 292-307.

Jaynes, E. T. (2003). Probability Theory: The Logic of Science.. Cambridge, UK: Cambridge University Press.

Joyce, J. M. (2007). Epistemic Deference: the Case of Chance. Proceedings of the Aristotelian Society, CVII(2), 187-206.

Keynes, J. M. (1921). A Treatise on Probability. London: Macmillan.

Lewis, D. (1980). A Subjectivist's Guide to Objective Chance. In R. C. Jeffrey (Ed.) Studies in Inductive Logic and Probability, vol. II. Berkeley: University of California Press. 
Lewis, D. (1994). Humean Supervenience Debugged. Mind, 103, 473-490.

List, C., \& Pivato, M. (2015). Emergent Chance. The Philosophical Review, 124(1), 119-152.

Nissan-Rozen, I. (2017). On the Inadmissibility of Some Historical Information. Philosophy and Phenomenological Research.

Pettigrew, R. (2012). Accuracy, Chance, and the Principal Principle. Philosophical Review, 121(2), 241-275.

Pettigrew, R. (2014). Accuracy, Risk, and the Principle of Indifference. Philosophy and Phenomenological Research.

Thau, M. (1994). Undermining and Admissibility. Mind, 103, 491-504.

Williamson, J. (2010). In Defence of Objective Bayesianism. Oxford: Oxford University Press. 